\title{
Esculetin reduces leukotriene B4 level in plasma of rats with adjuvant-induced arthritis
}

\author{
Przemysław Rzodkiewicz ${ }^{1,2}$, Emilia Gąsińska3 ${ }^{3}$ Michał Gajewski ${ }^{4}$, Magdalena Bujalska-Zadrożny³, \\ Dariusz Szukiewicz ${ }^{1}$, Sławomir Maśliński ${ }^{1}$ \\ ${ }^{1}$ Department of General and Experimental Pathology, CEPT Laboratory, Medical University of Warsaw, Poland \\ 2Department of Gerontology and Public Health, National Institute of Geriatrics, Rheumatology and Rehabilitation, Warsaw, Poland \\ ${ }^{3}$ Department of Pharmacodynamics, CEPT Laboratory, Medical University of Warsaw, Poland \\ ${ }^{4}$ Department of Biochemistry and Molecular Biology, National Institute of Geriatrics, Rheumatology and Rehabilitation, Warsaw, Poland
}

\begin{abstract}
Objectives: Esculetin (6,7-dihydroxycoumarin) is a natural coumarin with anti-oxidant, anti-inflammatory and anti-nociceptive activity. It acts as a potent inhibitor of lipoxygenases (5-LOX and 12-LOX) and decreases the production of matrix metalloproteinases (MMP-1, MMP-3 and MMP-9). Because both inhibition of lipoxygenases and inhibition of matrix metalloproteinases are effective strategies in the treatment of rheumatoid arthritis, we investigated whether esculetin may be effective in adjuvant-induced arthritis in rats.

Material and methods: The study was performed on male Lewis rats, in the adjuvant-induced arthritis model. Rats were divided into two groups: control (treated with $1 \%$ methylcellulose) and experimental (treated with esculetin $-10 \mathrm{mg} / \mathrm{kg}$ ip.). The tested compound was administered for 5 consecutive days starting on the $21^{\text {st }}$ day after induction of arthritis. Each group consisted of 7 animals. After 5 days of treatment, rats were anesthetized. The concentration of leukotriene B4 (LTB4) in plasma was determined by a competitive enzyme immunoassay.

Results: The LTB4 level in plasma of rats with adjuvant-induced arthritis is increased in comparison to rats without inflammation ( $362 \pm 34$ vs. $274 \pm 15$ pg/ml, $p<0.01$, respectively). Five-day treatment with esculetin in adjuvant-induced arthritis rats decreases the LTB4 level to a level comparable with rats without inflammation $(284 \pm 23 \mathrm{pg} / \mathrm{ml}, p<0.01)$.

Conclusions: LTB4 is the most potent chemotactic agent influencing neutrophil migration into the joint. It is known that its level in serum of patients with active rheumatoid arthritis is increased and correlates with disease severity. Some other lipoxygenase inhibitors have already been tested as potential drug candidates in clinical and preclinical trials for rheumatoid arthritis (Zileuton, PF-4191834). Because esculetin decreases the LTB4 level in plasma of rats in adjuvant-induced arthritis, it may also be considered as an attractive drug candidate for patients with rheumatoid arthritis.
\end{abstract}

Key words: esculetin, treatment, adjuvant-induced inflammation, leukotriene B4.

\section{Introduction}

Esculetin is a coumarin derivative of natural origin which occurs in numerous medicinal plants such as Aescullus hippocastanum (Sapindaceae), Artemisia capillaris (Asteraceae), Calendulla officinalis (Asteraceae), and
Viburnum opulus (Adoxaceae). These herbs have long been used in the treatment of pain, inflammation, and edemas in herbal medicine. Esculetin itself possesses antioxidant [1], anti-inflammatory [2] and antinociceptive [3] properties, among others, which are related to its inhibitory actions towards 5-LOX [4], 15-LOX [5] and

Address for correspondence:

Przemysław Rzodkiewicz, Department of Rheumatology and Public Health, National Institute of Geriatrics, Rheumatology and Rehabilitation, Spartanska 1, 02-637 Warsaw, Poland, e-mail: przemyslaw@rzodkiewicz.eu

Submitted: 23.08.2016; Accepted: 30.08.2016 
matrix metalloproteinases (MMPs) [6]. Due to its pharmacological properties esculetin may be considered as an attractive drug candidate for the treatment of rheumatoid arthritis.

In our previous study, we identified antinociceptive properties of esculetin in a non-inflammatory and acute inflammatory model of pain in rats [3]. The aim of this study was to determine whether esculetin may be useful in the treatment of adjuvant-induced arthritis in rats. Possible ways in which esculetin may modulate the course of the disease include inhibition of lipoxygenases, inhibition of neutrophil migration and activation, prevention of apoptosis and antioxidant action.

\section{Material and methods}

Animals: The study was conducted on male LEWIS rats (LEW/cmd), weighing 200-300 g. Animals were housed in a room maintained at $20 \pm 2^{\circ} \mathrm{C}$ under a $12 \mathrm{~h}$ light-dark cycle and had free access to food and water. The Second Ethical Committee for Experiments on Small Animals, Medical University of Warsaw, agreed to the tests being performed and accepted the experimental protocol.

The adjuvant-induced arthritis model (AIA) was induced in rats by subplantar injection of $100 \mu \mathrm{l}$ of Complete Freud Adjuvant into the left hind paw. Over the next days, inflammation involves more joints, and after 21 days it can be regarded as chronic (Fig. 1) [7]. The test compound was administered to the animals once a day for five successive days starting on the $21^{\text {st }}$ day. On the $25^{\text {th }}$ day after the induction of inflammation, $1.5 \mathrm{~h}$ after treatment, animals were anesthetized.

Drugs: Esculetin (ESC) and indomethacin were purchased from Sigma-Aldrich. The compound was dissolved in a $1 \%$ solution of methylcellulose and administered intraperitoneally (ip.) at a dose of $10 \mathrm{mg} / \mathrm{kg}$. The control group and group without inflammation received a 1\% solution of methylcellulose ip. Rats were anesthetized with ketamine (87 mg/kg ip.) and xylazine (14 mg/kg ip.)
(Biowet Puławy). Blood from the heart was collected into heparinized probes (BD cat no. 367526). To prevent ex vivo formation of eicosanoids, indomethacin $(10 \mu \mathrm{M})$ was added immediately after blood collection. Plasma probes were separated and stored at $-80^{\circ} \mathrm{C}$ for further LTB4 level measurement.

Leukotriene B4 (LTB4) immunoassay: LTB-4 level was measured in rat plasma samples. Before the assay, samples were purified from proteins by precipitation with ethanol. After centrifugation (3000x g, $10 \mathrm{~min}$ ) the supernatant was moved to a clean glass tube and evaporated. Next, samples were resuspended with an ELISA Buffer kit. Each sample was measured in two dilutions in duplicate. The measurement was performed with a Cayman Chemical LTB4 EIA Kit (item no. 520111), which is based on competitive binding of free LTB4 in the sample and tracer (titrated LTB4 labeled with acetylcholinesterase) to specific anti-LTB4 antiserum. Complexes bind to monoclonal antibodies attached to the test well. The reaction between acetylcholinesterase-LTB4 complexes labeled with monoclonal antibodies on the test well and Ellman's reagent produces 5-thio-2-nitrobenzoic acid, which has strong absorbance. Absorbance at 405 $\mathrm{nm}$ wavelength was measured with a Labtech 4000 LS microplate reader and is inversely proportional to the amount of LTB4 in the sample.

Data analysis: Analysis of differences of mean ranks between groups was performed with a one-tailed Mann-Whitney U-test. The significance of all tests was set at $p<0.05$. All statistical analyses were performed using Statistica v.12 (StatSoft, Kraków, Poland).

\section{Results}

Each sample was assayed in duplicate, and the ratio of the absorbance to that of the maximum binding $\left(B_{1} / B_{0}\right)$ was calculated for each sample and was within the expected range $(20 \%<x<80 \%)$. Linear regression was used to determine the equation and identify concentrations corresponding to each measurement. The concentration
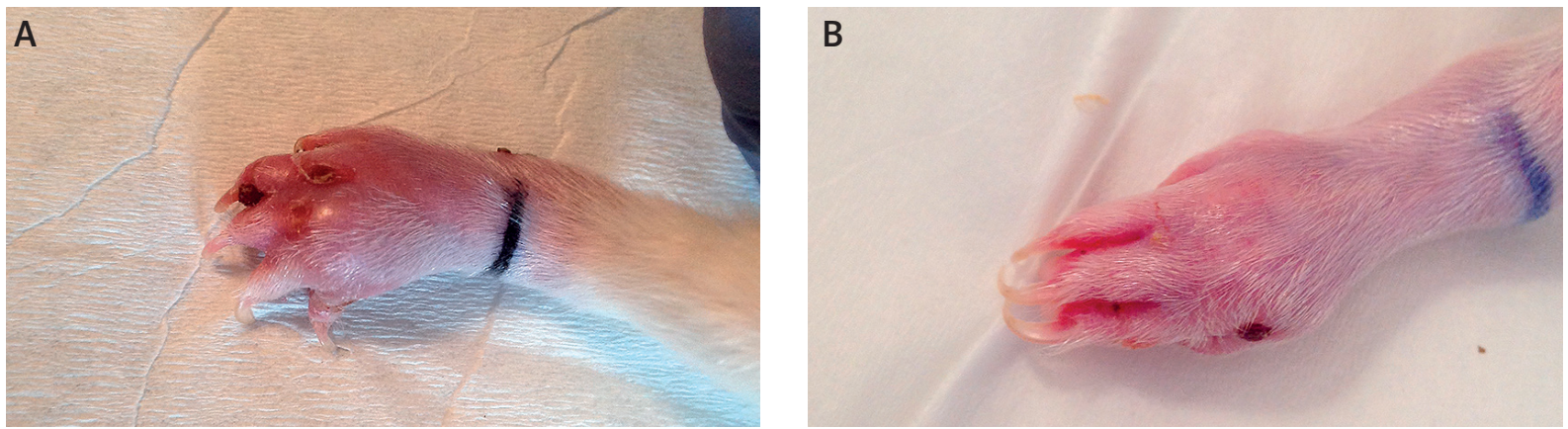

Fig. 1. Swelling of the ankle joints in rats with adjuvant-induced inflammation. A) Control group; B) Group treated with esculetin (10 mg/kg ip.). 
Table I. Level of LTB4 [pg/ml] in plasma of rats. Parameters were evaluated in the plasma of rats collected 90 min after treatment with the last dose of esculetin or methylcellulose

\begin{tabular}{|cccccccccccc|}
\hline Group & $N$ & Me & Q1 & Q3 & D2 $(X)$ & Min. & Max. & $m$ & SD & P1 & P2 \\
\hline Control & 7 & 272.90 & 260.69 & 278.07 & 235 & 252.41 & 300.81 & 272.91 & 15.34 & $X$ & 0.002 \\
\hline AlA-Control & 7 & 361.76 & 349.34 & 402.98 & 1185 & 335.27 & 432.18 & 374.96 & 34.42 & 0.002 & $X$ \\
\hline AlA-Esculetin & 7 & 274.41 & 266.21 & 306.17 & 547 & 263.51 & 326.41 & 284.24 & 23.40 & 0.443 & 0.002 \\
\hline
\end{tabular}

Control - group without inflammation; AlA-Control - control group with adjuvant-induced inflammation; AlA-Esculetin - group with adjuvant-induced inflammation treated with esculetin (10 mg/kg ip.); $N$ - number of cases; Me - median; Q1 - quartile 1; Q3 - quartile 3; $D 2(X)$ - variance; Min. - minimum; Max. - maximum; $m$ - average; SD - standard deviation; $P 1$ - $p$-value in comparison to control group; $P 2-p$-value in comparison to control group with adjuvant-induced inflammation.

of LTB4 in plasma of rats with adjuvant-induced arthritis was calculated as the average of two different dilutions $(1: 1$ and $1: 2)$ from each sample of blood - the difference between dilutions did not exceed $10 \%$. Due to the small number of animals in groups, non-parametric tests were used for statistical analysis. LTB4 level in plasma of rats with AIA was 33\% higher than in rats without inflammation (Mann-Whitney U-test; $U=0.00 ; Z=-3.066608$; $p=0.002165)$. In the group of rats treated for 5 days with esculetin $(10 \mathrm{mg} / \mathrm{kg}$ ip.) the level of LTB4 in plasma was lower than in the control group with AIA (Mann-Whitney U-test; $U=0.00 ; Z=3.066608 ; p=0.002165$ ) and was equal to the control group without inflammation (Mann-Whitney U-test; $U=18.00 ; Z=0.766652$; $p=0.443289$ ). Detailed data are presented in Table I.

\section{Discussion}

In this study, we demonstrated that esculetin reduces the LTB4 level in blood of rats with AIA. So far, not many studies have evaluated esculetin as a possible treatment for RA. In the course of RA proinflammatory cytokines such as TNF- $\alpha$, IL-1 $\beta$ and IFN- $\gamma$ induce apoptosis of chondrocytes, which weakens the cartilage significantly, contributing to the joint damage. The aim of treatment in RA is to inhibit tissue destruction. Several studies have shown that esculetin can act on chondrocytes to inhibit the secretion of metalloproteinases. However, it is not known if it affects the survival of these cells [8, 9]. Yamada et al. [6] found that esculetin inhibits matrix degradation in rabbit joint cartilage explants through the suppression of MMP synthesis, secretion, or activity. Possible mechanisms of this were described by Watanabe et al. [8], who found that esculetin could inhibit pro-matrix metalloproteinase-1/interstitial pro-collagenase and pro-matrix metalloproteinase-3/prostromelysin 1. Also Elliot et al. [9] noted that esculetin is an effective inhibitor of cartilage resorption. It was shown that this compound potently reduces proteoglycan and collagen loss and collagenolytic activity in resorbing cartilage. It has been demonstrated that these effects are related to reduced expression of matrix metalloproteinases: MMP-1,
MMP-3, and MMP-13 [9]. Because esculetin in addition to a decrease in MMP expression also has other mechanisms of action, these may not be the only mechanisms involved in the beneficial effects of esculetin.

Another pathological process involved in RA pathogenesis that may be modulated by esculetin is infiltration of neutrophils into the joints. Neutrophil mobilization accompanies active stages of RA and is an important factor damaging joints. Activated neutrophils are a source of free radicals and promote synthesis of matrix metalloproteinases that destroy joint tissue [1012]. It seems that lipoxygenases are crucial enzymes involved in neutrophil migration. Different strategies to reduce neutrophil migration and chemotaxis were shown to be effective. It was observed that in 5-LOX knockout mice neutrophil migration induced by zymosan injection was reduced [13]. A similar effect was observed with the lipoxygenase inhibitor MK886 and the leukotriene B4 receptor (BLT1) blocker CP105696 [13]. MK886 reduced neutrophil migration into the knee joint of mice with antigen-induced arthritis [14]. Esculetin is a potent inhibitor of lipoxygenases involved in the synthesis of LTB4, which is a powerful chemotactic agent influencing neutrophil migration into the joint [15]. So far, esculetin has not been tested in animal models of arthritis, but studies with this compound in other models have shown that it possesses antioxidant activity [1], and it reduces neutrophil infiltration in animal models of inflammation [16].

Because esculetin reduces the LTB4 level in the blood, it may possibly modulate neutrophil migration in arthritis, but further research is needed to address this issue. It was shown that the level of LTB4 in the serum of patients with active RA is increased and correlates with disease severity $[17,18]$. Critical involvement of LTB4 in arthritis induction and severity was confirmed in studies with a mouse K/BxN serum transfer model of arthritis [19]. It was also observed that 5-LOX inhibitors weaken TNF- $\alpha$-induced inflammation in human synovial fibroblasts [20]. Some 5-LOX inhibitors have already been tested in preclinical and clinical studies, with promising results. The selective 5 -LOX inhibitor zileuton inhibited 
LTB4 production by $70 \%$ in patients with $\mathrm{RA}$ in a randomized double blind placebo-controlled study [21]. The lipoxygenase inhibitor PF-4191834 developed by Pfizer was able to reduce pain and inflammation in an adjuvant-induced arthritis model in rats [22].

\section{Conclusion}

Our observations confirm that esculetin may effectively modulate the LTB4 level in adjuvant-induced arthritis in rats and may be considered as an attractive drug candidate for patients with rheumatoid arthritis.

\section{Acknowledgements}

This project was financed by a grant from the National Science Center based on decision no. DEC-2011/03/N/ NZ4/03765. Research subject implemented with CePT infrastructure funded by the EU - the European Regional Development for 2007-2013.

The authors declare no conflict of interest.

\section{References}

1. Martin-Aragon S, Benedi JM, Villar AM. Effects of the antioxidant (6,7-dihydroxycoumarin) esculetin on the glutathione system and lipid peroxidation in mice. Gerontology 1998; 44: 21-25.

2. Witaicenis A, Seito LN, Di Stasi LC. Intestinal anti-inflammatory activity of esculetin and 4-methylesculetin in the trinitrobenzenesulphonic acid model of rat colitis. Chem Biol Interact 2010; 186: 211-218.

3. Rzodkiewicz P, Gasinska E, Maśliński S, et al. Antinociceptive properties of esculetin in non-inflammatory and inflammatory models of pain in rats. Clin Exp Pharmacol Physiol 2015; 42: 213-219.

4. Du L, Zhang Z, Luo X, et al. Binding investigation of human 5-lipoxygenase with its inhibitors by SPR technology correlating with molecular docking simulation. J Biochem 2006; 139: 715-723.

5. Yokota S, Oda T, Fahimi HD. The role of 15-lipoxygenase in disruption of the peroxisomal membrane and in programmed degradation of peroxisomes in normal rat liver. J Histochem Cytochem 2001; 49: 613-622.

6. Yamada H, Watanabe K, Saito T, et al. Esculetin (dihydroxycoumarin) inhibits the production of matrix metalloproteinases in cartilage explants, and oral administration of its prodrug, CPA926, suppresses cartilage destruction in rabbit experimental osteoarthritis. J Rheumatol 1999; 26: 654-662.

7. Nagakura Y, Okada M, Kohara A, et al. Allodynia and hyperalgesia in adjuvant-induced arthritic rats: time course of progression and efficacy of analgesics. J Pharmacol Exp Ther 2003; 306: 490-497.

8. Watanabe K, Ito A, Sato T, et al. Esculetin suppresses proteoglycan metabolism by inhibiting the production of matrix metalloproteinases in rabbit chondrocytes. Eur J Pharmacol 1999; 370: 297-305.
9. Elliott $\mathrm{S}$, Rowan AD, Carrere $\mathrm{S}$, et al. Esculetin inhibits cartilage resorption induced by interleukin 1alpha in combination with oncostatin M. Ann Rheum Dis 2001; 60: 158-165.

10. Gajewski M, Rzodkiewicz P, Maśliński S. Aktualne poglądy na znaczenie neutrofilów w reumatoidalnym zapaleniu stawów. Wciąż neutrofile czy może już mikrofagi? Reumatologia 2011; 49: 344-350.

11. Leppert D, Hauser SL, Kishiyama JL, et al. Stimulation of matrix metalloproteinase-dependent migration of T cells by eicosanoids. FASEB J 1995; 9: 1473-1481.

12. Ahluwalia N, Lin AY, Tager AM, et al. Inhibited aortic aneurysm formation in BLT1-deficient mice. J Immunol 2007; 179: 691697.

13. Guerrero AT, Verri WA Jr., Cunha TM, et al. Involvement of LTB4 in zymosan-induced joint nociception in mice: participation of neutrophils and PGE2. J Leukoc Biol 2008; 83: 122-130.

14. Grespan R, Fukada SY, Lemos HP, et al. CXCR2-specific chemokines mediate leukotriene B4-dependent recruitment of neutrophils to inflamed joints in mice with antigen-induced arthritis. Arthritis Rheum 2008; 58: 2030-2040.

15. Henderson WR, Jr. The role of leukotrienes in inflammation. Ann Intern Med 1994; 121: 684-697.

16. Uberti AF, Olivera-Severo D, Wassermann GE, et al. Pro-inflammatory properties and neutrophil activation by Helicobacter pylori urease. Toxicon 2013; 69: 240-249.

17. Ahmadzadeh N, Shingu M, Nobunaga M, et al. Relationship between leukotriene B4 and immunological parameters in rheumatoid synovial fluids. Inflammation 1991; 15: 497-503.

18. Gursel T. Firat S, Ercan ZS. Increased serum leukotriene B4 level in the active stage of rheumatoid arthritis in children. Prostaglandins Leukot Essent Fatty Acids 1997; 56: 205-207.

19. Chen M, Lam BK, Kanaoka Y, et al. Neutrophil-derived leukotriene B4 is required for inflammatory arthritis. J Exp Med 2006; 203: 837-842.

20. Lin HC, Lin TH, Wu MY, et al. 5-Lipoxygenase inhibitors attenuate TNF-alpha-induced inflammation in human synovial fibroblasts. PLoS One 2014; 9: e107890.

21. Weinblatt ME, Kremer JM, Coblyn JS, et al. Zileuton, a 5-lipoxygenase inhibitor in rheumatoid arthritis. J Rheumatol 1992; 19: 1537-1541.

22. Masferrer JL, Zweifel BS, Hardy M, et al. Pharmacology of PF-4191834, a novel, selective non-redox 5-lipoxygenase inhibitor effective in inflammation and pain. J Pharmacol Exp Ther 2010; 334: 294-301. 